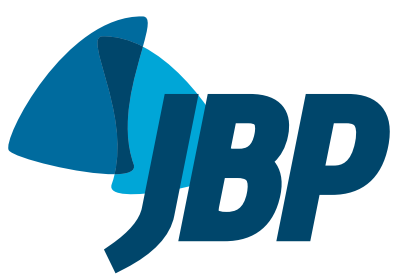

\title{
A storm of stones in the lungs: an uncommon sequela of varicella pneumonia
}

\author{
Gaetano Rea1, Adriano Costigliola², Cecilia Calabrese ${ }^{2}$
}

A 29-year-old female patient, never smoker, restorer by profession, presented with slight malaise and the flu that lasted for a few days. An X-ray of the chest showed various nodules of varying sizes in random distribution and with high density in both lungs, similar to calcifications (Figure 1A). The laboratory test results were unremarkable. Pulmonary function tests and blood gases were normal: $\mathrm{FEV}_{1}=3.23 \mathrm{~L}$ ( $97 \%$ of predicted); $\mathrm{FVC}=3.81 \mathrm{~L}(98 \%$ of predicted); $\mathrm{DLCO}=98 \% ; \mathrm{pO}_{2}=97 \mathrm{mmHg}$; and $\mathrm{pCO}_{2}=39$ $\mathrm{mmHg}$. An HRCT of the chest was performed to clarify the chest X-ray findings, showing that the totality of nodules was calcified, but there were no calcified lymph nodes (axial HRCT scan with mediastinal window; Figure 1B). The HRCT scans also showed numerous small bilateral nodules-sharply defined and randomly distributed in both lungs-but no interstitial thickening or any other pathologic findings (Figures $1 \mathrm{C}$ and 1D). Parathyroid function, autoantibodies, and quantiFERON-TB (Cellestis, Ltd., Carnegie, Australia) testing was negative, and blood calcium levels were normal. The patient confirmed a severe varicella infection in childhood (at age 4 years), and antibody testing for the varicella-zoster virus showed positive results ( $\mathrm{IgG}=348 \mathrm{mIU} / \mathrm{mL}$ and $\mathrm{IgM}=0.34 \mathrm{mIU} /$ $\mathrm{mL}$ ). Varicella (chickenpox) is a contagious viral disease transmitted by respiratory droplets. The development of multiple, small, diffuse nodular calcifications in both lungs with noncalcified lymph nodes is an uncommon sequela of varicella pneumonia.

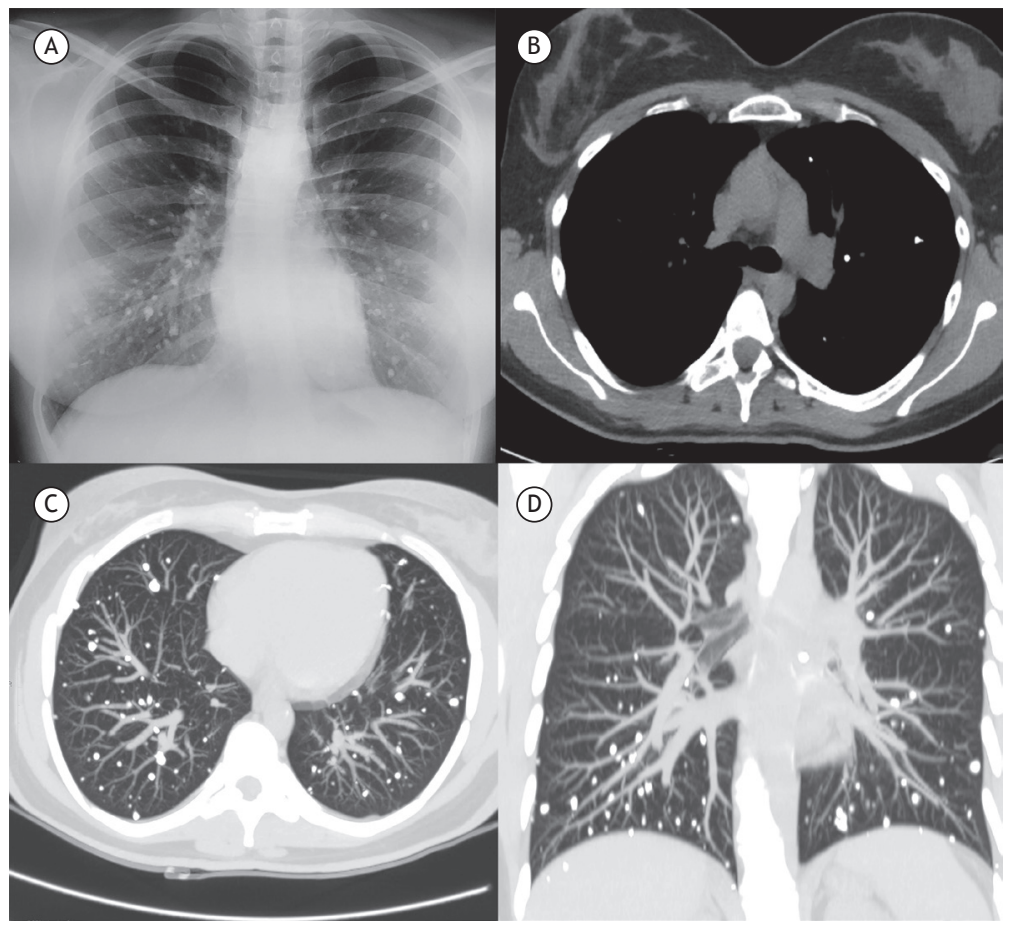

Figure 1. In $A$, anteroposterior chest $X$-ray showing innumerable, coarse and punctate micronodules with high calcific density throughout both lungs; in B, axial HRCT scan (mediastinal window) showing no mediastinal calcified lymph nodes. Axial (in C) and coronal (in D) HRCT scans of the chest (thickness of maximum intensity projection: $14 \mathrm{~mm}$ and $30 \mathrm{~mm}$, respectively) showing scattered, randomly distributed calcified nodules in both lungs, with no calcified mediastinal lymph nodes, suggestive of healed varicella pneumonia in the appropriate clinical setting.

\section{RECOMMENDED READING}

. Zanetti G, Hochhegger B, Marchiori E. Calcified multinodular lung lesions: a broad differential diagnosis. Clin Respir J. 2015 Jun 16. [Epub ahead of print] https://doi.org/10.1111/crj.12338

2. Marchiori E, Souza AS Jr, Franquet T, Müller NL. Diffuse high-attenuation pulmonary abnormalities: a pattern-oriented diagnostic approach on high-resolution CT. AJR Am J Roentgenol. 2005;184(1):273-82. https:// doi.org/10.2214/ajr.184.1.01840273

3. Amin SB, Slater R, Mohammed TL. Pulmonary calcifications: a pictorial review and approach to formulating a differential diagnosis. Curr Probl Diagn Radiol. 2015;44(3):267-76. https://doi.org/10.1067/J.cpradiol.2014.12.005 